\title{
Sudden cardiac death in mitral valve prolapse: are we ready for risk stratification?
}

Volume 9 Issue 2 - 2017

\section{Introduction}

The association of mitral valve prolapse (MVP) with ventricular arrhythmias and sudden cardiac death (SCD) has been recognized for few decades. ${ }^{1-6}$ Nevertheless, until recently this association has been mainly a vague one; with not much of an evidence to estimate its prevalence, clarify if this is a mere association or a causative effect, understand the mechanism underlying this association and hence risk stratify these patients. Accordingly, the management of a young patient with mitral valve prolapse complaining of atypical chest pain, non specific palpitations or a Holter recording of ventricular premature beats (VPC) continues to engage a major challenge for the practicing physician. Moreover, many of us are aware of MVP patients who were timely diagnosed and apparently well treated but still died in a devastating way resulting from absence of appropriate risk stratification to help prevent arrhythmic events.

The data collected until recently was based on autopsy studies of SCD victims in whom MVP was the only pathologic cardiac finding, pointing for a specific subgroup of MVP patients, particularly women of younger age without significant MR as a risk group for SCD. ${ }^{1}$ Otherwise, population-based studies looking into the natural history of asymptomatic MVP patients revealed an elevated 10year all-cause mortality rate, predicted by significant MR and low LVEF; similar to the all-cause mortality reported in severe MR patients with a flail leaflet. ${ }^{2,3}$ Nevertheless, these studies pointed for a subgroup of MVP patients experiencing SCD who were typically young and asymptomatic; many of whom had a preserved LVEF and no significant MR. Notably, although significant MR was found to predict ventricular arrhythmia among MVP patients in some studies ${ }^{4,7}$ this was not shown by others. ${ }^{1-3,8}$

During the last years emerging data has come shedding light on specific risk factors for arrhythmia in MVP patients. A review of Myo Clinic's out of hospital cardiac arrest cases including 1200 cases, revealed 24 SCD cases which were defined as idiopathic after ruling out ischemia, various cardiomyopathy or channelopathies. ${ }^{9}$ Ten out of these 24 idiopathic SCD cases were found to have bileaflet MVP, of whom $90 \%$ were women; $80 \%$ had biphasic or inverted $\mathrm{T}$ waves on ECG and $100 \%$ had a significant ventricular premature activity on a Holter monitoring. Accordingly, a malignant MVP subgroup was characterized as young women with bileaflet MVP, and frequent complex ventricular ectopy. Importantly, the complex ventricular ectopy was characterized by activity originating from both LV outflow tract and LV papillary muscles. ${ }^{9}$ Notably, bileaflet MVP by itself without the other risk profile was shown to predict a reduced risk for SCD. ${ }^{10}$

Thereafter, a more detailed and specific arrhythmic risk factors emerged from a recent study, based on the Italian pathology registry of SCD victim's hearts, reviewing 43 SCD cases in which MVP was the only pathology. ${ }^{11}$ The study found bileaflet MVP to exist in $70 \%$ of those cases, LV papillary muscle fibrosis in all cases and

\author{
Rav Acha M, Wolak A \\ Cardiology Department, Shaare Tzedek Hospital, Israel
}

Correspondence: Rav Acha M, Cardiology Department, Shaare Tzedek Hospital, Jerusalem, Israel,

Email ravacha.moshe@gmail.com

Received: June 27, 2017 | Published: July 10, 2017

inferobasal fibrosis in $88 \%{ }^{11}$. These findings were then confirmed by comparing CMR findings of MVP patients with and without ventricular arrhythmias, revealing LV late-gadolinium enhancement (LGE) in $93 \%$ and $14 \%$ of MVP patients with ventricular ectopy, correspondingly. Importantly, the CMR LGE distribution was overlapping the histopathology fibrosis of SCD hearts. Thus, a regional LV papillary muscle and inferobasal fibrosis was found to characterize both MVP patients with SCD as well as MVP patients with complex ventricular ectopy; presenting a novel specific risk factor for SCD among MVP patients. Notably, similar findings on CMR or histopathology have been previously reported. ${ }^{12,13}$ Given the correlation between the source of ventricular ectopy and the areas of cardiac fibrosis, a mechanical stretch hypothesis has been raised, ${ }^{14}$ by which a mechanical stretch of the myocardium in these specific areas results from the prolapsing MV leaflets. Accordingly, multiple mitral annular and leaflet pathological morphologies were suggested to explain the above mechanical stretch. Among these were mitral annular dysjunction, defined as the separation between the LA-valve junction and the atrial aspect of the LV free wall, as well as posterior systolic curling defined as unusual systolic motion of the posterior mitral ring on the adjacent myocardium.

Assuming mitral valve pathology in MVP patients contributes to cardiac fibrosis and hence cardiac arrhythmias, the effect of MV repair surgery on reduction of arrhythmia was examined. Previous anecdotal reports suggested mitral repair as an effective measure to control arrhythmias. ${ }^{15,16}$ Furthermore, a recent study including 8 patients with bileaflet MVP implanted with ICD due to ventricular malignant arrhythmia, revealed a significant reduction in malignant arrhythmia following surgical correction. ${ }^{17}$ Nevertheless, a more recent study from the same center, including a cohort of 32 consecutive patients that underwent MV surgery for mitral regurgitation secondary to bileaflet MVP who had available pre- and post-operative Holter monitoring data, demonstrated that mitral valve surgery did not uniformly reduce the burden of ventricular arrhythmias in patients with bileaflet MVP. ${ }^{18}$ 
Thus, the role of MV surgery to reduce malignant arrhythmia is not conclusive and further studies will be needed before this could be advocated.

\section{Conclusion}

In summary, multiple publications have pointed for an increased risk for SCD among relatively young and otherwise healthy MVP patients. Weather this correlation presents a mere association or a causative effect is still unknown. Moreover, the contribution of significant MR for ventricular arrhythmia is controversial. Recent data has shed light on a subgroup of MVP patients which seem at risk for SCD including young females with bileaflet MVP with complex ventricular ectopy originating from LV outflow of LV papillary muscles. Further studies enforced this data by revealing fibrosis in these specific locations among MVP-SCD victims or MVP patients with malignant arrhythmias, thus elucidating for a structuralfunctional relation for malignant arrhythmias in MVP. Accordingly, it seems that it is time to consider a CMR for risk stratification of these patients.

\section{Acknowledgements}

None.

\section{Conflicts of interest}

Author declares there are no conflicts of interest.

\section{Funding}

None.

\section{References}

1. Dollar AL, Roberts WC. Morphologic comparison of patients with mitral valve prolapse who die suddenly with patients who die from severe valvular dysfunction or other conditions. $J$ Am coll cardiol. 1991;17(4):921-931.

2. Avierinos JF, Gersh BJ, Melton LJ, et al. Natural history of asymptomatic mitral valve prolapse in the community. Circ. 2002;106:1355-1361.

3. Grigioni F, Entriquez-sarano M, Ling LH, et al. Sudden death in mitral regurgitation due to flail leaflet.J Am Coll cardiol. 1999;34(7):2078-2085.

4. Turker Y, Ozaydin M, Acar G, et al. Predictors of ventricular arrhythmias in patients with mitral valve prolapse. Int $J$ cardiovasc imaging. 2010;26(2):139-145.
5. Stefanadis C, Toutouzas P. Mitral valve proplapse: the merchant of Venice or much ado about nothing? E Heart J . 2000;21(4):255-288.

6. Chugh SS, Kelly KL, Titus JL. Sudden cardiac death with apparently normal heart. Circ. 2000;102(6):649-654.

7. Narayanan K, UY-Evanado A, Teodorescu C, et al. Mitral valve prolapse and sudden cardiac arrest in the community. Heart Rhythm. 2016;13(2):498-503.

8. Vohra J, Sathe S, Warren R, et al. Malignant ventricular arrhythmias un patients with mitral valve prop[lase and mild mitral regurgitation. Pacingg Clin Electophysiol. 1993;16(3 Pt 1):387-393.

9. Siram CS, Syed FF, Ferguson ME, et al. Malignant bileaflet mitral valve prolapse syndrome in patients with otherwise idiopathic out of hospital cardiac arrest. J am Coll Cardiol. 2013;62(3):222-230.

10. Nordhues BD, Siontis KC, Nkomo Vt, et al. Bileaflet mitral valve prolapse and risk of ventricular dysrhythmias and death. $J$ cardiovasc electrophysiol. 2016;27(4):463-468.

11. Basso C, Perazzolo Marra M, Rizzo S, et al. Arrhythmic mitral valve prolapse and sudden cardiac death. Circ. 2015;132(7):556-566.

12. Han Y, peters DC, salton CJ, et al. Cardiovascular magnetic resonance characterization of mitral valve prolapse. $J$ am Coll cardiol . 2008;1(3):294-303.

13. La Vecchia L, Ometto R, Centonfante P, et al. Arrhythmic profile, ventricular function, and histomorphometric findings in patients with idiopathic ventricular tachycardia and mitral valve proplase: clinical and prognostic evaluation. Clin cardiol. 1998;21(10):731-735.

14. Perazzolol Marra M, Basso C, De Lazzari M, et al. Morphofunctional abnormalities of mitral annulus and arrhythmic mitral valve proplase. Circ Cardiovasc Imaging. 2016;9(8):1-10.

15. Abbaadi DR, Purbey R, Poornima IG. Mitral valve repair is an effective treatment for ventricaulr arrhythmias in mitral valve prolapsed syndrome. In J cardiol. 2014;177(1):E16-E18.

16. Pocok WA, Barlow JB, Marcus RH, et al. Mitral valvuloplasty for lifethreatening ventricular arrhythmias in mitral valve proplase. Am Heart J. 1991;121(1 Pt 1):199-202.

17. Vaidya VR, DeSimone CV, Damle N, et al. Reduction in malignant ventriucular arrhythmia and appropriate shocks following surgical correction of bileaflet mitral valve prolapse. J Interv Card electrophysiol. 2016;46(2):137-143.

18. Naksuk N, syed FF, Krittanawong C, et al. The effect of mitral valve surgery on ventricular arrhythmia in patients with bileaflet mitral valve prolapse. Indian Pacing Electophysiol J . 2016;16(6):187-191. 